\title{
Tuning surface plasmon-exciton coupling via thickness dependent plasmon damping
}

\author{
Sinan Balci, ${ }^{1}$ Coskun Kocabas, ${ }^{2}$ Simge Ates, ${ }^{2}$ Ertugrul Karademir, ${ }^{2}$ Omer Salihoglu, ${ }^{2}$ and Atilla Aydinli ${ }^{2, *}$ \\ ${ }^{1}$ Department of Mechanical Engineering, University of Turkish Aeronautical Association, 06790 Ankara, Turkey \\ ${ }^{2}$ Department of Physics, Advanced Research Laboratories, Bilkent University, 06800 Ankara, Turkey
}

(Received 1 August 2012; published 4 December 2012)

\begin{abstract}
In this paper, we report experimental and theoretical investigations on tuning of the surface plasmon-exciton coupling by controlling the plasmonic mode damping, which is defined by the plasmonic layer thickness. The results reveal the formation of plasmon-exciton hybrid state characterized by a tunable Rabi splitting with energies ranging from 0 to $150 \mathrm{meV}$. Polarization-dependent spectroscopic reflection measurements were employed to probe the dispersion of the coupled system. The transfer matrix method and analytical calculations were used to model the self-assembled $J$-aggregate/metal multilayer structures in excellent agreement with experimental observations.
\end{abstract}

DOI: 10.1103/PhysRevB.86.235402

PACS number(s): 73.20.Mf, 42.50.Pq, 71.35.-y

\section{INTRODUCTION}

Controlling light-matter interaction plays a fundamental role in emerging optical phenomena, which have broad applications in optoelectronics, nonlinear optics, lasers, and spectroscopy. Cavity quantum electrodynamics (QED) provides a quantum mechanical description for these fundamental interactions in confined geometries. ${ }^{1-5}$ When an atom is placed in an optical cavity, the resonant modes of the cavity are coupled with electronic transitions of the atom. Trapped ions in high- $Q$ cavities, ${ }^{1} Q$ dots in photonic crystal cavities, ${ }^{2}$ and organic materials with excitonic transitions in optical cavities $^{3}$ can be understood within the framework of cavity QED. Excitonic materials placed in a plasmonic cavity provide a new platform to study cavity QED. ${ }^{5-8}$ Coupling between surface plasmons (SPs) on the metal surfaces ${ }^{6}$ and excitons in optical materials results in a coupled quantum system. ${ }^{7-14}$ Strong coupling ${ }^{15}$ between excitons and SPs is needed to form a coupled hybrid state, which arises when an exciton mode resonates with a plasmon mode. ${ }^{8}$ Strong coupling in plasmon-exciton coupling has been demonstrated in a variety of plasmonic systems bearing localized SPs ${ }^{13}$ or propagating SPs. ${ }^{14}$ The strength of coupling is characterized by comparing the rate of energy transfer between the matter and the cavity and the decay rates of the individual states. When the latter is significantly smaller than the former, a strong coupling regime is established. ${ }^{15}$ An important indication of strong coupling is the anticrossing of the exciton and plasmon modes, where, in the absence of a strong coupling, they would have crossed. The enhanced electromagnetic field of SPs and strong oscillator strength of excitons can provide the necessary conditions for strong coupling.

The ability to tune the SP-exciton coupling would make this system a promising candidate for a range of new applications..$^{7-17}$ The strength of this interaction determines both the absorption and emission properties of molecules placed near plasmonic structures, effectively governing the optical properties of such a hybrid system. As a measure of coupling, Rabi splitting in SP-exciton systems is given by $\hbar \Omega_{R}=\sqrt{4 V^{2}-\left(\gamma_{p}-\gamma_{e}\right)^{2}}$, where $V$ is the coupling parameter, $\gamma_{p}$ and $\gamma_{e}$ are the linewidth (i.e., damping) of the bare plasmons and excitons, respectively. ${ }^{8}$ Rabi splitting can be tuned in two ways: (i) by varying the excitonic properties of the matterlike component (i.e., changing $V$ or $\gamma_{e}$ ) or (ii) by controlling the properties of the plasmonic system (i.e., varying $\left.\gamma_{p}\right)$. The former has been demonstrated by varying the optical density of the excitonic system ${ }^{9,14}$ on flat metal surfaces. Very recently, Schwartz et al. have demonstrated reversible switching between the weak and strong coupling regimes via a photochemical process, which changes the strength of the dipole moment. ${ }^{16}$ In this work, we study tuning of SP-exciton coupling by only controlling the damping of the plasmonic component. We were able to tune the Rabi-splitting energies ranging from 0 to $150 \mathrm{meV}$ by varying the plasmonic layer thickness, which defines the damping of the plasmonic mode. We studied dispersion of the coupled system analytically as well as using transfer matrix method in excellent agreement with the experimentally extracted data.

\section{EXPERIMENTAL DETAILS}

Figure 1(a) shows the experimental setup used to probe the SP-exciton coupling. ${ }^{18} \mathrm{We}$ used excitons of a cyanine dye [5,5',6,6'-tetrachloro-di-(4-sulfobutyl) benzimidazolocarbocyanine, TDBC, from Few Chemicals; see Fig. 1(a) for the chemical structure of a TDBC molecule], forming selfassembled nanostructures at a high concentration called $J$ aggregate, as a matter component because of its strong dipole moment ${ }^{19,20}$ and a thin film of silver to support SPs. The formation of self-assembled nanostructures of $J$ aggregate at high concentration can be identified by narrowing and redshifting of the absorption spectrum. The measured absorption linewidth of excitons, $\gamma_{e}$, is approximately $51 \mathrm{meV}$ and peaked at the exciton resonant energy of $2.09 \mathrm{eV}$. Polyvinyl alcohol (PVA) was dissolved in water by heating the water-PVA suspension at $150{ }^{\circ} \mathrm{C}$ for half an hour. ${ }^{3,19,20}$ TDBC molecules were dissolved in water and then mixed with $1.5 \%$ PVA solution in a 1:1 (volume-to-volume) ratio to obtain $0.75 \%$ PVA and known concentration of TDBC molecules in water. The solution was then spin coated at 3000 rpm onto metal-evaporated glass substrates to build opticalquality films. Wavelength-dependent optical constants $(n, k)$ of TDBC molecules as a function of TDBC concentration in the PVA matrix were calculated from the experimental results obtained by using a variable-angle spectroscopic ellipsometer (VASE). The refractive index of transparent materials is often 
(a)

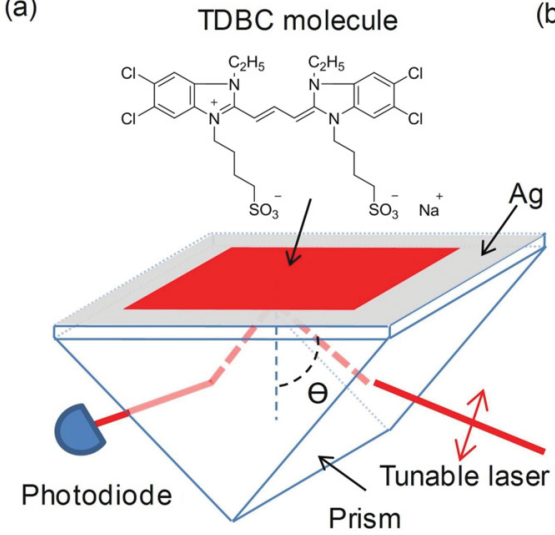

(b)

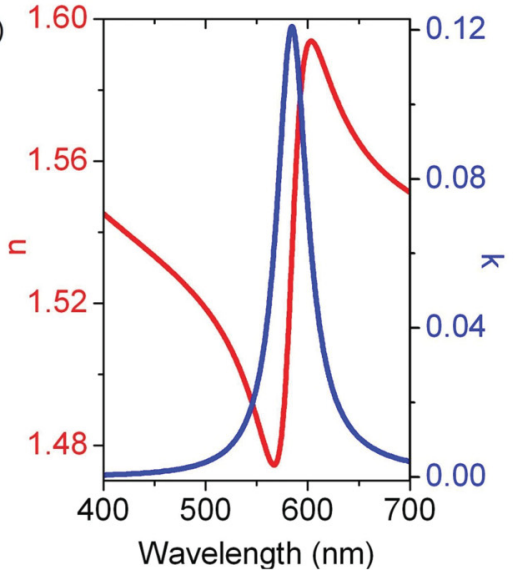

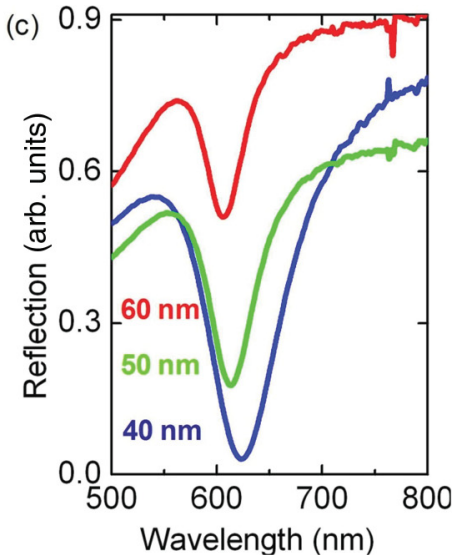

FIG. 1. (Color online) (a) Schematic representation of KC, where the prism increases the momentum of the incident light used to obtain polaritonic dispersion curves. Tunable and polarized incident laser light excites SPs on silver metal. The silicon photodiode measures the intensity of the reflected laser light. The chemical structure of the shown molecule is chemical structure of a single TDBC molecule. Measured optical constants $n$ and $k$ (b) of aggregated TDBC molecules in PVA matrix. (c) Plasmon resonance reflection spectra as a function of wavelength for various metal film thicknesses.

described using the Cauchy relationship, which is typically described as $n(\lambda)=A+B / \lambda^{2}+C / \lambda^{4}$, where the three terms are adjusted to fit the refractive index of the transparent materials as a function of wavelength to the experimental data. However, when the material is not transparent, the Lorentz oscillator model should be used to describe the optical constants of the material. The Lorentz oscillator model is written as $\varepsilon=\varepsilon_{1}+A E_{c} /\left(E_{c}^{2}-E^{2}-i B E\right)$, where $A, B$, $E_{c}, \varepsilon_{1}$ are the amplitude, the broadening, the peak energy, and the background dielectric constant, respectively. The spectroscopic ellipsometer is used to measure the complex refractive index of PVA films and TDBC containing PVA films as a function of the wavelength of the light for a given incidence angle. The effective medium approximation model was used to calculate the optical constants of the TDBC/PVA blend films on Si substrates from the experimentally obtained data. In this model, Cauchy and Lorentz models are coupled with each other. Thicknesses of the prepared films were found to be in the range of 300 and $360 \mathrm{~nm}$. Figure 1(b) shows the experimentally obtained optical constants of thin film of TDBC molecules dispersed in the PVA polymeric matrix. In this case, TDBC molecules were dissolved in 5\% PVA in water and spin coated at $3000 \mathrm{rpm}$ onto a silicon substrate in order to measure optical constants of TDBC molecules in the PVA matrix.

\section{RESULTS AND DISCUSSIONS}

Plasmon-exciton coupling is monitored through the polarization-dependent reflectivity measurements from the silver surface in the Kretschmann configuration (KC) [Fig. 1(a)] by tilting the sample at an angle of $\theta$ and recording how the reflectivity of the sample changes with the wavelength of the incident light, which is $p$ polarized to excite SPs at the $\mathrm{Ag} / \mathrm{J}$ aggregate interface, as shown in the experimental part. The samples are mounted on a prism with index-matching liquid to maintain the optical index continuity between the prism and the glass substrate. ${ }^{18,21}$ The reflectivity goes to a minimum, where the phase-matching condition between the incident light and
SP is satisfied, Fig. 1(c). This is achieved when the horizontal component of the incident light $\left(k_{x}\right)$ matches the real part momentum of SPs $\left(k_{s p}\right)$. The dispersion relation of SPs at a metal-dielectric interface can be defined as $k_{x}=k_{0} n_{p} \sin (\theta)=$ $k_{s p}=\frac{2 \pi}{\lambda} \sqrt{\frac{\varepsilon_{m} \varepsilon_{d}}{\varepsilon_{m}+\varepsilon_{d}}}$, where $\lambda$ is the wavelength of the incident light, $k_{0}$ is the free-space wave vector of the incident light, $\varepsilon_{m}$ and $\varepsilon_{d}$ are the dielectric constants of metal and dielectric, respectively, $n_{p}$ is the refractive index of the prism, and $\theta$ is the SP resonance angle. ${ }^{18,21}$ Figure $1(\mathrm{c})$ shows the reflection spectrum of a flat Ag surface through a prism for various thicknesses. The excitation of a SP on a flat metal surface through a prism [Fig. 1(c)] can be captured in the reflectivity spectrum with a Lorentzian line shape, $R(\omega) \propto \frac{\gamma_{p}}{\left(\omega-\omega_{0}\right)^{2}-\gamma_{p}^{2}}$. The damping term $\gamma_{p}$ determines the linewidth of the reflection spectrum. The linewidth of SP varies, $\gamma_{p}$, from 330 to $85 \mathrm{meV}$ as the thickness is changed from 30 to $70 \mathrm{~nm}$ [Fig. 1(c)].

The effect of plasmon damping on plasmon-exciton interaction energy was studied in this study by varying the plasmonic layer thickness and hence the linewidth of the SP resonance in a controlled manner. Measuring the polarization-dependent reflectivity (in KC) of PVA- (doped with TDBC) coated Ag surface with various thicknesses, allows us to probe plasmon-exciton coupling. The evolution of plasmon-exciton reflection curves with the thickness of the plasmonic layer is shown in Fig. 2(a). The separation between the polaritonic branches representing the Rabi-splitting energy increases with the thickness of the plasmonic layer [Figs. 2(b)-2(c)]. As the thickness of plasmonic layer increases, the lifetime of SPs increases, indicating that the damping of the plasmonic mode decreases, which results in an increase in SP-exciton interaction, hence large Rabi-splitting energies. The polaritonic dispersion formula can also be written by replacing the wave vector with the incidence angle. It should be noted here that since the wave vector $k=(2 \pi / \lambda) \sin (\theta)$ is not constant for a fixed incidence angle for the lower and upper polaritonic branches, the calculated Rabi-splitting values from the polariton reflection curves plotted in terms of wavelength as a function of the incidence angle is different from the energy 

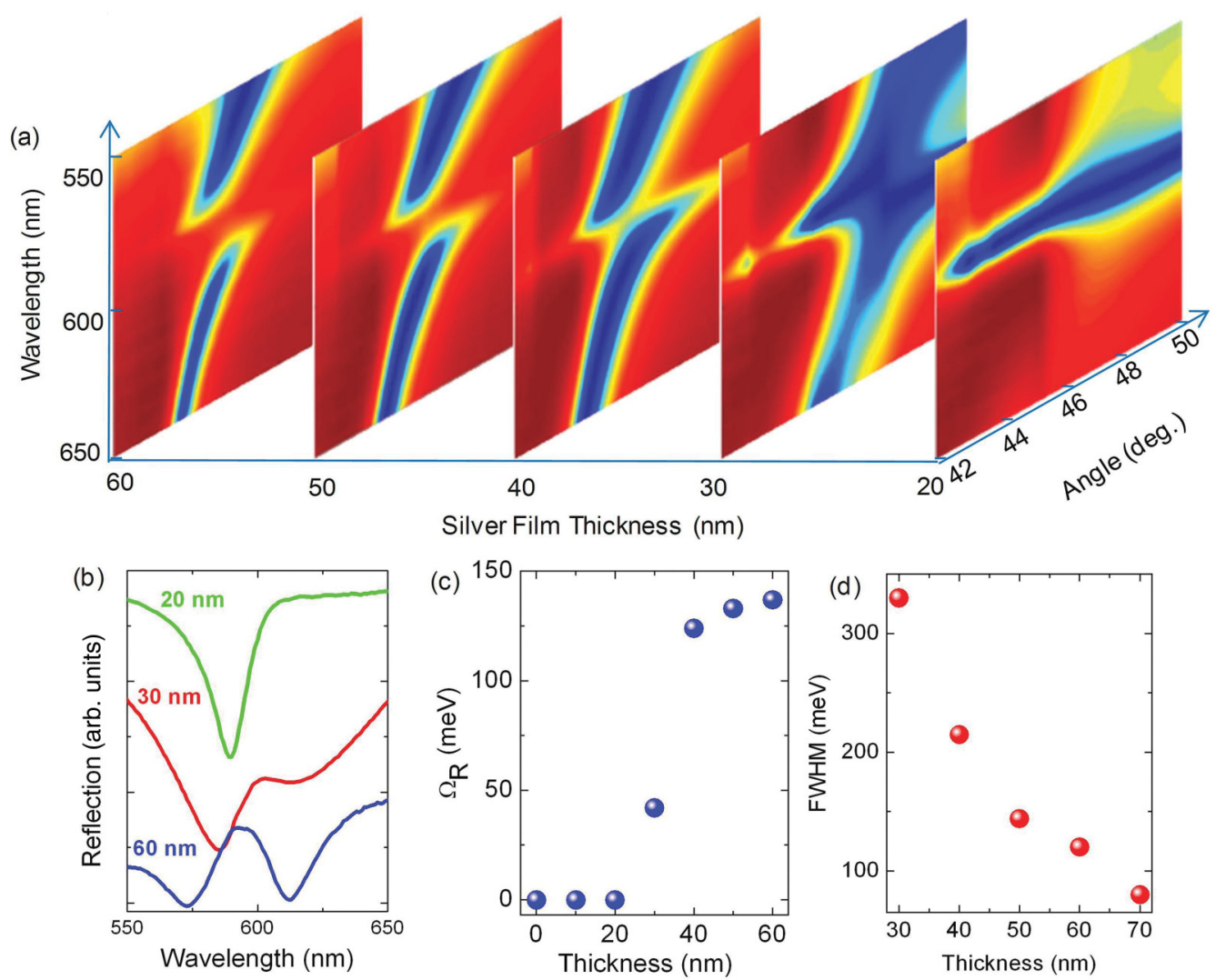

FIG. 2. (Color online) Plasmon-exciton coupling as a function of plasmonic layer thickness. (a) Evolution of polariton reflection curves with varying Ag film thickness. Concentration of TDBC molecules in the polymer matrix is constant (1.25 mM) for all the measurements. (b) Evolution of SP resonance reflection curves with the plasmonic layer thickness. (c) Rabi-splitting energy increases with the plasmonic layer thickness. (d) Full width at half-maximum (linewidth) of the plasmon resonance reflection curve decreases with the increase in the plasmonic layer thickness.

versus wave-vector polariton dispersion curves. ${ }^{22}$ During this study, we monitored relative change in the Rabi-splitting energy and calculated the Rabi-splitting energies from the wavelength versus angle polariton reflection curves. In order to understand why the Rabi-splitting energy increases with the plasmonic layer thickness, we obtained SP resonance reflection spectra of bare plasmonic layers and calculated the linewidth of the SP resonance reflection spectra [Fig. 2(d)], which indicated a decrease in the linewidth of the SP peak with the plasmonic layer thickness.

The dipole moment of TDBC molecules on the silver surface interacts with the electric field of SPs. The coupling energy between the SP and the TDBC molecules is governed by the formula, $E=\vec{\mu} \bullet \overrightarrow{\mathrm{E}}$, where $\vec{\mu}$ is the dipole moment of the material and $\overrightarrow{\mathrm{E}}$ is the electric field generated by the plasmons. ${ }^{8}$ The rate of energy transfer between the SP and the exciton can be expressed as $g=E / \hbar$. At resonance, the two normal modes of the coupled oscillator, in the absence of damping, are given as $s_{ \pm}=A \cos \left(\omega_{e x} \pm g\right) t$ in which $g=V_{0} / \hbar$ is the rate of energy transfer and $\omega_{e x}$ is the excitonic transition frequency. ${ }^{8}$ Given the plasmon mode damping $\gamma_{p}$ and the exciton damping $\gamma_{e}$, the two normal modes become damped oscillations in time expressed as $S_{ \pm}=A e^{-(\kappa+\gamma) t / 2} \cos \left(\omega_{e x} \pm\right.$ $\left.g^{\prime}\right) t$, where $g^{\prime}=\sqrt{\left(\frac{V_{0}}{\hbar}\right)^{2}-\frac{1}{4}\left(\gamma_{p}-\gamma_{e}\right)^{2}}$. It is obvious that matching $\gamma_{p}$ and $\gamma_{e}$ maximizes the coupling and, therefore, the
Rabi-splitting energy. ${ }^{8}$ We measured damping of SPs, $\gamma_{p}$, and damping of the excitons, $\gamma_{e}$, using reflectivity [Fig. 1(c)] and ellipsometric measurements [Fig. 1(b)], respectively. Since the linewidth of the plasmon resonance is proportional to the plasmon mode damping and inversely proportional to the SP lifetime, the change in the linewidth affects the energy transfer rate and hence the Rabi-splitting energy. Using the coupled oscillator model, the energies of the polaritonic branches of the coupled oscillator system can be defined as $E_{1,2}(k)=$ $\left[E_{s p}(k)+E_{e x}\right] / 2 \pm \frac{1}{2}\left(\sqrt{\left(\hbar \Omega_{R}\right)^{2}+\left(E_{s p}(k)-E_{e x}\right)^{2}}\right)$, where $k$ is the in-plane-wave vector, $E_{1}$ and $E_{2}$ are the energies of the upper and lower polaritonic states, $E_{e x}$ is the energy of the bare excitons, $E_{s p}$ is the energy of noninteracting or bare plasmon mode, $\hbar \Omega_{R}$ is the Rabi-splitting energy $\left(\hbar \Omega_{R}=2 V_{0}\right.$ in which $V_{0}$ is the plasmon-exciton interaction energy occurring at the momentum at which energy splitting between the polaritonic states reaches a minimum ${ }^{8}$ ).

In order to monitor the evolution of the polariton reflection curves with the optical density of the TDBC molecules, reflection dips as a function of the light incidence angle was acquired, Fig. 3. At the Rabi-splitting angle, where bare exciton and bare plasmon energies overlap, their reflection spectra demonstrate an increase in the separation between the lower and upper polariton branches as a function of $J$-aggregate concentration. Plotting the square root of the TDBC molecule 

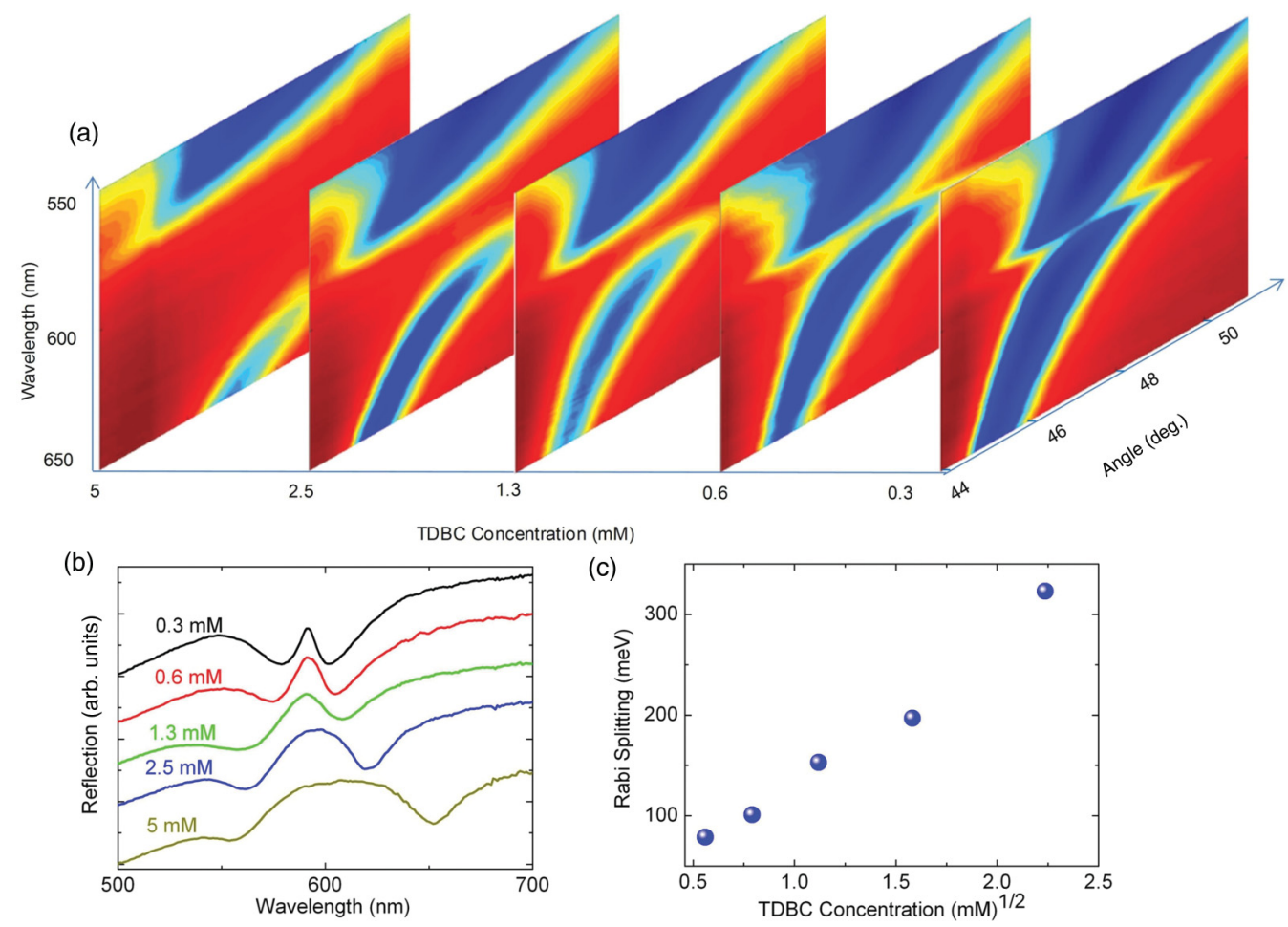

FIG. 3. (Color online) Plasmon-exciton coupling as a function of TDBC concentration. (a) Evolution of polariton reflection curves with varying concentration of TDBC molecules in the PVA matrix. As the concentration of the TDBC molecules increases in the PVA matrix, plasmon-exciton coupling energy or Rabi-splitting energy increases. (b) Polariton reflection curves of thin Ag films containing active layer of varying concentration of TDBC molecules in the PVA matrix. (c) Rabi-splitting increases linearly with the square root of the TDBC concentration in the PVA matrix.

concentration in the PVA matrix versus Rabi-splitting energy confirms the expected linear dependence ${ }^{7,14}$ (Fig. 3). It is obvious that Rabi-splitting energy increases linearly with the square root of TDBC concentration. ${ }^{14}$ Enhancement of Rabi splitting with the concentration of TDBC molecules is due to the increase in the optical density of $J$-aggregate film and hence the increase in the total oscillator strength. For a specific absorbing material, Rabi splitting between the plasmonic state and the excitonic state is expected to vary as $\left(\alpha_{0} L\right)^{1 / 2,7}$ where $\alpha_{0}$ is the peak absorption coefficient and $L$ is the absorbing material film thickness. The data shown in Fig. 3 represent a clear anticrossing of the two hybrid peaks, which is characteristic of the strong coupling when the bare SP mode and the bare TDBC exciton resonance mode overlap. ${ }^{15}$ The blue and red regions show reflectivity minima and maxima, respectively. ${ }^{18,21}$ It is worth mentioning here that without the TDBC molecules placed on the plasmonic layer, the SP reflection curve does not show any anticrossing peaks, which are characteristic of SP-exciton coupling.

Using experimentally obtained optical constants of the $J$-aggregate-polymer layer [Fig. 1(b)] and plasmon mode damping for various metal film thicknesses [Fig. 1(c)], the
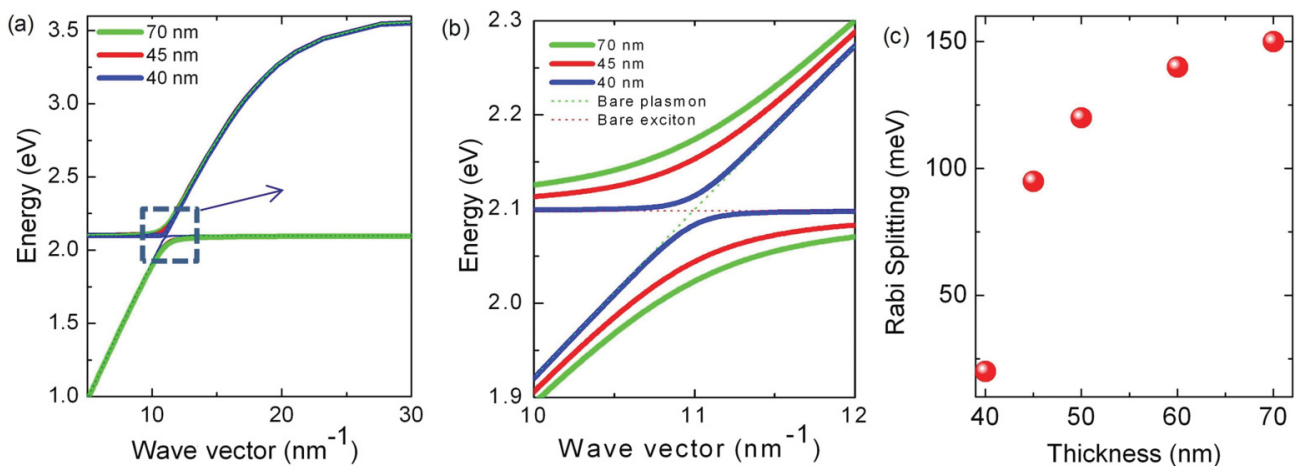

FIG. 4. (Color online) Analytically calculated plasmon-exciton coupling. (a) Calculated polariton dispersion relation as a function of the linewidth of the SP resonance using experimental data in reference. ${ }^{23}$ (b) Zoom in the polaritonic dispersion curve in (a). The dotted red and green lines show the bare exciton and bare plasmon lines, respectively. (c) Rabi-splitting energy obtained from the analytically calculated data in (b) increases with the thickness of the plasmonic layer. 

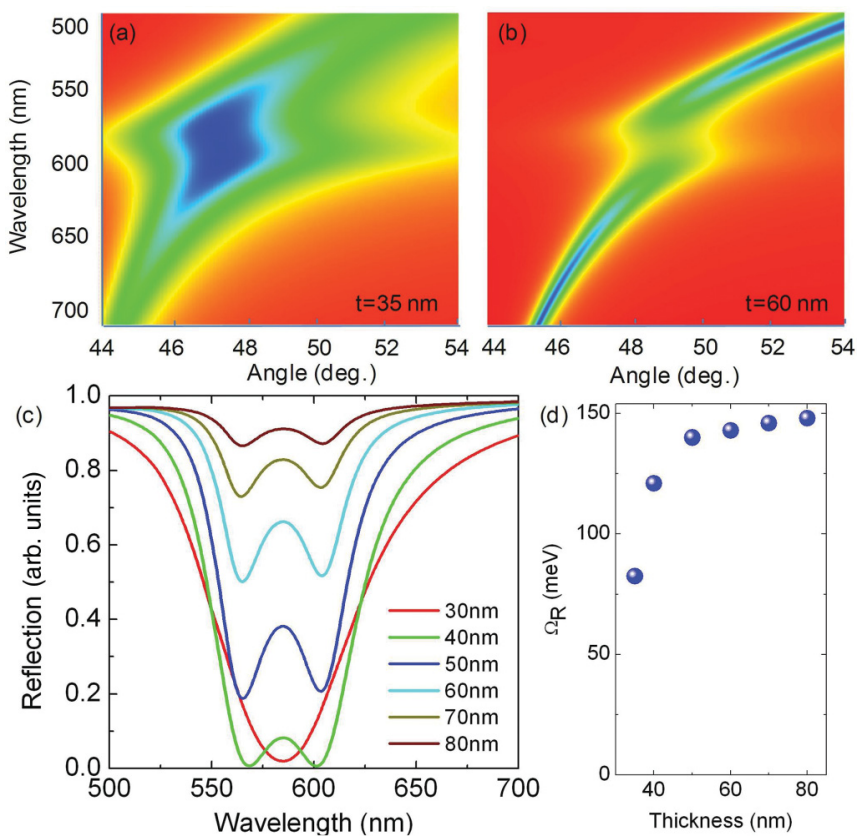

FIG. 5. (Color online) Using transfer matrix method, calculated polariton reflection curves and reflection spectra for varying plasmonic layer thickness. (a) Calculated polariton reflection curve for 35-nm-thick Ag film. Optical density of TDBC molecules is fixed. The blue and red regions show the reflectivity minima and maxima, respectively. (b) Calculated polariton reflection curve for 60 -nm-thick Ag film. (c) Evolution of polariton reflection spectra as a function of plasmonic layer thickness. (d) Calculated Rabi-splitting energies from the polariton reflection curves.

polariton dispersion curves of the coupled state were analytically calculated using the equation for the coupled state as $E_{1,2}(k)=\left[E_{s p}(k)+E_{e x}\right] / 2 \pm \frac{1}{2}\left(\sqrt{\left(\hbar \Omega_{R}\right)^{2}+\left(E_{s p}(k)-E_{e x}\right)^{2}}\right)$ and shown in Fig. 4. Figure 4(c) shows Rabi-splitting energies as a function of plasmonic layer thickness. Since, in this case, exciton damping is constant; Rabi splitting can be tuned by only varying the plasmon mode damping. In addition, SP-exciton coupling can be understood within the framework of classical electromagnetic theory. We calculated the dispersion relation of the SP-exciton system by solving Maxwell's equations for each layer using the transfer matrix method. ${ }^{3,8,17}$ Such a model with experimentally obtained optical constants of $J$-aggregate film and plasmonic layer provides a classical explanation of the SP-exciton coupling. Figures 5(a) and 5(b) show the calculated reflectivity maps for $J$-aggregate film fabricated from a 5-mM TDBC in PVA solution onto 35- and 60-nm-thick silver layers, respectively. It is worth mentioning, here, that optical constants of 5-mM TDBC in PVA were experimentally measured in a spectroscopic ellipsometer [Fig. 1(b)] and inserted in these calculations. Figure 5(c) shows the reflection spectrum at an angle of $43^{\circ}$ for various metal film thicknesses. The small difference between the reflection curves in Fig. 5(c) and the experimental reflection curves shown in Fig. 2(b) may be due to (i) the small difference between the SP resonance data in reference $^{23}$ and the experimental SP resonance data in this work or (ii) possible small deviations in metal film thickness measurements. The calculated Rabi splitting as a function
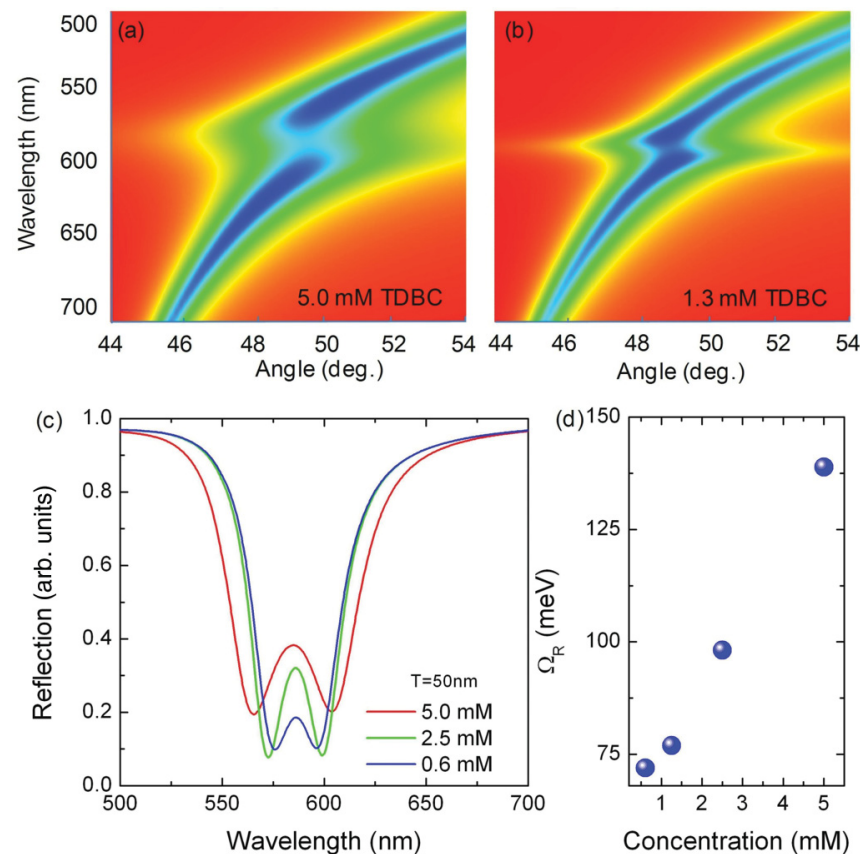

FIG. 6. (Color online) Using transfer matrix method, calculated polariton reflection curves and reflection spectra for varying optical density of TDBC molecules and a fixed plasmonic layer thickness. (a) Calculated polariton reflection curve for 5.0-mM TDBC molecules in the PVA matrix. The blue and red regions show the reflectivity minima and maxima, respectively. (b) Calculated polariton dispersion curve for 1.3-mM TDBC molecules in the PVA matrix. (c) Polariton reflection curves as a function of the TDBC concentration on 50-nm-thick plasmonic layer. (d) Calculated Rabi-splitting energies as a function of the TDBC concentration.

of the metal film thickness is given in Fig. 5(d). The Rabi splitting increases when the thickness of the plasmonic layer increases. The Rabi-splitting values we obtain using transfer matrix method are in excellent agreement with the analytical calculations described above in Fig. 4 and experimental observations shown in Fig. 2. Furthermore, using the transfer matrix method, polariton reflection curves and reflection spectra for varying optical density of TDBC molecules and a fixed plasmonic layer thickness are calculated and shown in Fig. 6. The calculated polariton reflection curves for 5.0- and 1.3-mM TDBC molecules in the PVA matrix are shown in Fig. 6(a) and Fig. 6(b), respectively. Polariton reflection curves as a function of the TDBC concentration on 50-nm-thick plasmonic layer clearly shows the increase in Rabi-splitting energies with the increase in the TDBC concentration. ${ }^{7,14}$ The theoretical results shown in Figs. 4-6 are in excellent agreement with the experimental results demonstrated in Figs. 2 and 3.

\section{CONCLUSION}

In conclusion, we have experimentally and theoretically studied the tunability of plasmon-exciton coupling on $J$-aggregate-metal surfaces. Polarization-dependent spectroscopic reflection measurements were used to probe SP-exciton coupling. The results demonstrate the formation of plasmonexciton hybrid states, characterized by large Rabi-splitting 
energies, which can be tuned either by varying the optical density of the TDBC molecules or by varying the damping of the SP. The magnitude of the coupling depends on both the exciton oscillator strength and the plasmon mode damping. Optical constants of thin films of $J$-aggregated TDBC molecules dispersed in PVA polymeric matrix were measured using a spectroscopic ellipsometer. The detailed experimental and theoretical results presented in this study open the way for efficient energy transfer and coherent coupling in plasmonic devices at nanoscale dimensions, and it will help in understanding the fundamental aspects of SP-exciton coupling and designing hybrid plasmonic devices with superior performances. We note that recent demonstration of controlling plasmon loss via temperature ${ }^{24}$ or tuning the crystallinity of the plasmonic layer $^{25}$ or by optical pumping ${ }^{25,26}$ can also be applied to tune plasmon-exciton coupling.

\section{ACKNOWLEDGMENTS}

This work has been supported by Grants No. 110T790, and No. 110T589 from the Scientific and Technological Research Council of Turkey (TUBITAK). *aydinli@fen.bilkent.edu.tr

${ }^{1}$ M. Brune, F. Schmidt-Kaler, A. Maali, J. Dreyer, E. Hagley, J. M.

Raimond, and S. Haroche, Phys. Rev. Lett. 76, 1800 (1996).

${ }^{2}$ T. Yoshie, A. Scherer, J. Hendrickson, G. Khitrova, H. M. Gibbs,

G. Rupper, C. Ell, O. B. Shchekin, and D. G. Deppe, Nature (London) 432, 200 (2004).

${ }^{3}$ D. G. Lidzey, D. D. C. Bradley, A. Armitage, S. Walker, and M. S. Skolnick, Science 288, 1620 (2000).

${ }^{4}$ D. G. Lidzey, D. D. C. Bradley, T. Virgili, A. Armitage, M. S. Skolnick, and S. Walker, Phys. Rev. Lett. 82, 3316 (1999).

${ }^{5}$ Y. Zhu, D. J. Gauthier, S. E. Morin, Q. Wu, H. J. Carmichael, and T. W. Mossberg, Phys. Rev. Lett. 64, 2499 (1990).

${ }^{6}$ W. L. Barnes, A. Dereux, and T. W. Ebbesen, Nature (London) 424, 824 (2003).

${ }^{7}$ D. E. Gomez, K. C. Vernon, P. Mulvaney, and T. J. Davis, Nano Lett. 10, 274 (2010).

${ }^{8}$ J. R. Tischler, M. S. Bradley, Q. Zhang, T. Atay, A. Nurmikko, and V. Bulovic, Org. Electron. 8, 94 (2007).

${ }^{9}$ T. K. Hakala, J. J. Toppari, A. Kuzyk, M. Pettersson, H. Tikkanen, H. Kunttu, and P. Torma, Phys. Rev. Lett. 103, 053602 (2009).

${ }^{10}$ J. Dintinger, S. Klein, F. Bustos, W. L. Barnes, and T. W. Ebbesen, Phys. Rev. B 71, 035424 (2005).

${ }^{11}$ Y. B. Zheng, B. K. Juluri, L. L. Jensen, D. Ahmed, M. Lu, L. Jensen, and T. J. Huang, Adv. Mater. 22, 3603 (2010).

${ }^{12}$ G. A. Wurtz, P. R. Evans, W. Hendren, R. Atkinson, W. Dickson, R. J. Pollard, and A. V. Zayats, Nano Lett. 7, 1297 (2007).
${ }^{13}$ N. T. Fofang, T. H. Park, O. Neumann, N. A. Mirin, P. Nordlander, and N. J. Halas, Nano Lett. 8, 3481 (2008).

${ }^{14}$ C. Bonnand, J. Bellessa, and J. C. Plenet, Phys. Rev. B 73, 245330 (2006).

${ }^{15}$ J. Bellessa, C. Bonnand, J. C. Plenet, and J. Mugnier, Phys. Rev. Lett. 93, 036404 (2004).

${ }^{16}$ T. Schwartz, J. A. Hutchison, C. Genet, and T. W. Ebbesen, Phys. Rev. Lett. 106, 196405 (2011).

${ }^{17}$ J. B. Pendry and A. MacKinnon, Phys. Rev. Lett. 69, 2772 (1992).

${ }^{18}$ S. Balci, A. Kocabas, C. Kocabas, and A. Aydinli, Appl. Phys. Lett. 97, 131103 (2010).

${ }^{19}$ E. E. Jelley, Nature (London) 138, 1009 (1936).

${ }^{20}$ G. Scheibe, Angew. Chem. 49, 563 (1936).

${ }^{21}$ S. Balci, E. Karademir, C. Kocabas, and A. Aydinli, Opt. Lett. 36, 3401 (2011).

${ }^{22}$ C. Symonds, C. Bonnand, J. C. Plenet, A. Brehier, R. Parashkov, J. S. Lauret, E. Deleporte, and J. Bellessa, New J. Phys. 10, 065017 (2008).

${ }^{23}$ E. D. Palik, Handbook of Optical Constants of Solids (Academic Press, Boston, 1985).

${ }^{24}$ J. S. G. Bouillard, W. Dickson, D. P. O'Conner, G. A. Wurtz, and A. V. Zayats, Nano Lett. 12, 1561 (2012).

${ }^{25}$ G. A. Wurtz, R. Pollard, W. Hendren, G. P. Wiederrecht, D. J. Gosztola, V. A. Podolskiy, and A. V. Zayats, Nat. Nanotechnol. 6, 107 (2011).

${ }^{26}$ M. Ren, B. Jia, J. Y. Ou, E. Plum, J. Zhang, K. F. MacDonald, A. E. Nikolaenko, J. Xu, M. Gu, and N. I. Zheludev, Adv. Mater. 23, 5540 (2011). 